\title{
Robust band of critical states in time-reversal symmetry-broken fermionic systems with lattice selective disorder
}

\author{
Eduardo V. Castro* \\ Centro de Física das Universidades do Minho e Porto, 4710-057 Braga, Portugal; \\ Departamento de Física e Astronomia, Faculdade de Ciencias, Universidade do Porto, 4169-007 Porto, Portugal; \\ CeFEMA, Instituto Superior Técnico, Universidade de Lisboa, Avenida Rovisco Pais, 1049-001 Lisboa, Portugal; \\ and Beijing Computational Science Research Center, Beijing 100084, China \\ Raphael de Gail, M. Pilar López-Sancho $\odot$, and María A. H. Vozmediano \\ Instituto de Ciencia de Materiales de Madrid, CSIC, Sor Juana Inés de la Cruz 3, Cantoblanco, 28049 Madrid, Spain
}

(Received 25 June 2019; published 25 November 2019)

\begin{abstract}
We analyze the localization properties of two-dimensional systems based on partite lattices with a basis. Contrary to standard results, we find that a band of critical states emerges for systems in the unitary class A preserving spin symmetry when disorder is unevenly distributed over the basis atoms. The critical metal arises when the less disordered sublattice is connected and has time-reversal symmetry broken. The unexpected robustness to disorder presented here is an appealing result which can be measured in optical lattices.
\end{abstract}

DOI: 10.1103/PhysRevResearch.1.033129

\section{INTRODUCTION}

Anderson localization [1] is one of the best studied phenomena in modern condensed matter. The localization transition in $d=2$ depends critically on the symmetry class of the system [2]. In the standard symmetry classification completed with the addition of topological properties [3], the behavior of the unitary class (class A) was initially understood in the context of the quantum Hall effect, where the spin degree of freedom is still a good quantum number. It was established numerically that at the center of each Landau level band there is only one critical state, an extended state where the localization length diverges linearly with system size, indicative of a vanishing $\beta$ scaling function [4,5]. The case when both timereversal symmetry $\mathcal{T}$ and spin-rotation symmetry are broken has been the subject of recent investigation [6-9], and the physics was found to be different. It seems well established that a band of extended states, and not a single state, shows up. Depending on the model, this band can be made entirely of critical states [7,9] or can be a band of truly extended states $[6,7]$. Interestingly enough, the transition from the localized side to the critical metal at the energy $E_{c}$ is accompanied by a divergent localization length $\xi(E)$ of the form $\xi(E) \sim$ $\exp \left(\alpha / \sqrt{\left|E-E_{c}\right|}\right)$, reminiscent of a Berezinskii-KosterlitzThouless transition [10]. Critical metallic behavior has also been found in Weyl semimetals [11-13].

\footnotetext{
*eduardo.castro@tecnico.ulisboa.pt

Published by the American Physical Society under the terms of the Creative Commons Attribution 4.0 International license. Further distribution of this work must maintain attribution to the author(s) and the published article's title, journal citation, and DOI.
}

In this work we show examples of critical metallic behavior in class A systems with spin-rotation symmetry. The critical metal is found on lattices with a basis when disorder is unevenly distributed over the basis atoms. The minimal conditions over the less disordered sublattice for the occurrence of the critical metal are that it has to be connected and have broken time-reversal symmetry.

In all cases we will have a Hamiltonian defined on a lattice with two or more sublattices. Potential (Anderson) disorder is implemented by adding to the Hamiltonian the term $\sum_{i \in A, B} \varepsilon_{i} c_{i}^{\dagger} c_{i}$, with a uniform distribution of random local energies $\varepsilon_{i} \in[-W / 2, W / 2]$. For selective disorder we have disorder strength $W_{i}$ for sublattice $i$.

\section{MODELS AND RESULTS}

We will begin by summarizing the situation of the Haldane model [14], a prototype of the class A system with spinrotation symmetry. A robust metallic state has already been found by introducing strong selective disorder in one sublattice $[15,16]$. The tight-binding Hamiltonian can be written as

$$
H=-t \sum_{\langle i, j\rangle} c_{i}^{\dagger} c_{j}-t_{2} \sum_{\langle i, j\rangle\rangle} e^{-i \phi_{i j}} c_{i}^{\dagger} c_{j}+\Delta \sum_{i} \eta_{i} c_{i}^{\dagger} c_{i},
$$

where $c_{i}=A, B$ are defined in the two triangular sublattices that form the honeycomb lattice. The first term $t$ represents a standard real nearest-neighbor (NN) hopping that links the two triangular sublattices. The $t_{2}$ term represents a complex next-nearest-neighbor (NNN) hopping $t_{2} e^{-i \phi_{i j}}$ acting within each triangular sublattice with a phase $\phi_{i j}$ that has opposite signs $\phi_{i j}= \pm \phi$ in the two sublattices. This term breaks timereversal symmetry and opens a nontrivial topological gap at the Dirac points. We have done our calculations for the simplest case $\phi=\pi / 2$. The last term represents a staggered 

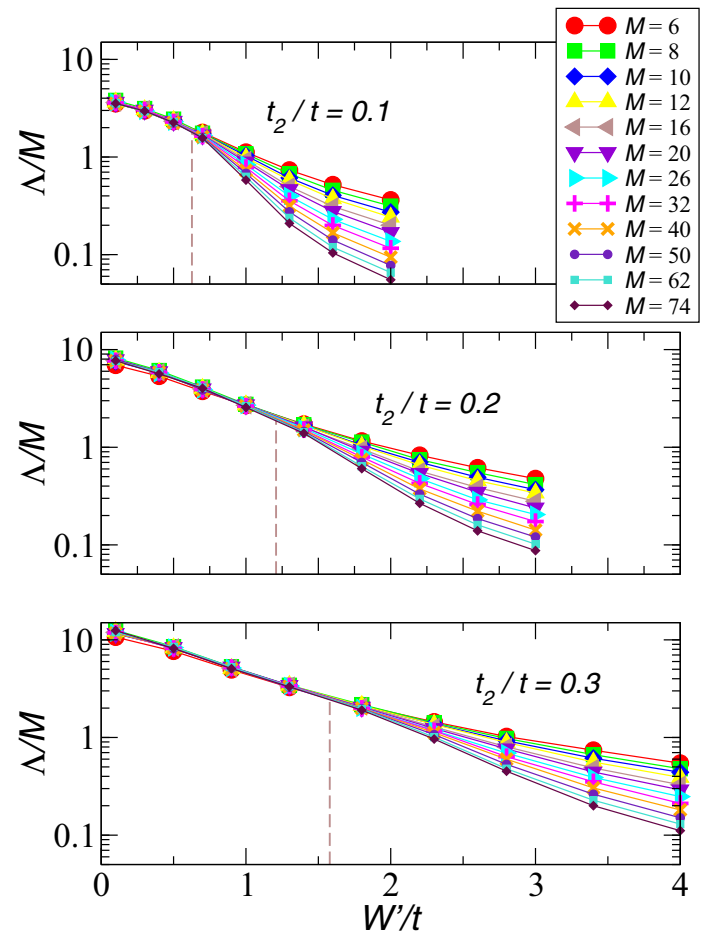

FIG. 1. Normalized localization length $\Lambda / M$ as a function of disorder strength $W_{B}$ in the less disordered sublattice for various NNN hoppings $t_{2}$, calculated for a long tube with $M$ unit cells in circumference. The vertical dashed line is a guide to the eye for the transition from critical to localized behavior. We have set the disorder $W_{A}=100 t$ and chosen the energy $E=-0.05 t$.

potential $\left(\eta_{i}= \pm 1\right)$. It breaks inversion symmetry and opens a trivial gap at the Dirac points. We use spinless fermions; taking spin into account amounts to a spin degeneracy factor and leads to the same physics.

We have shown previously [16] that by disordering a single sublattice, i.e., making $W_{A} \neq 0$ but keeping $W_{B}=0$, a band of critical states appears for $W_{A}>W_{A}^{c}$ and it is robust no matter how large the disorder is. This result has been established using both a level spacing statistical analysis and the transfer matrix method.

In order to explore the robustness of the metallic state, we study its localization behavior with a transfer matrix analysis. We set the disorder $W_{A}=100 t$ and study the metal-insulator transition as a function of $W_{B}$ for a given energy inside the band of critical states. We choose $E=-0.05 t$ and change the disorder strength $W_{B}$. The results are shown in Fig. 1, where we show the normalized localization length $\lambda / M$ as a function of disorder strength in the less disordered sublattice $W_{\mathrm{LD}}$, for various values of the NNN hopping $t_{2}$, calculated for a long tube with $M$ unit cells in circumference. It is obvious that there is a critical disorder strength $W_{\mathrm{LD}}^{c}$ below which $\lambda / M$ does not change with $M$, signaling the presence of a critical state. For $W_{\mathrm{LD}}>W_{\mathrm{LD}}^{c}$, the normalized localization length decreases with $M$, as expected for a localized state.

The results above indicate that two-dimensional class A systems do not always turn into an insulator (trivial or nontrivial) after levitation and pair annihilation have occurred between extended states. For systems with more than one sub- lattice and selective disorder, i.e., at least one sublattice with low disorder below some critical value while the remaining atoms have high disorder, the final state might be a metal. In what follows we try to identify the minimal requirements for such a state to occur.

The following are the main characteristics that we consider relevant for the physics presented in the case of the Haldane model. (i) The original system has bands with nontrivial Berry curvature. (ii) After strong selective disorder a "clean sublattice" with full connectivity remains. In the case of the Haldane model it is a triangular lattice with a complex NN hopping parameter. (iii) The remaining clean sublattice has complex hopping parameters that break time-reversal symmetry irrespective of the possible global topological triviality of the original lattice.

Next we will present alternative lattice models with a richer sublattice structure where we can discriminate the necessity of these ingredients.

Honeycomb lattice with enlarged unit cell models. In Ref. [16] we showed that the critical metal arises in the Haldane model irrespectively of whether the clean limit phase is trivial (nonzero Chern number) or topologically nontrivial. In any case, the triviality in that example was due to a cancellation of the Chern number upon integration of a nonzero Berry curvature. In order to explore the importance of the Berry curvature on the localization properties of the system, we study two different tight-binding models realized in the honeycomb lattice with a tripled unit cell. The basis vectors in real space read $\mathbf{a}_{1}=\frac{3 a}{2}(-\sqrt{3}, 1)$ and $\mathbf{a}_{2}=\frac{3 a}{2}(\sqrt{3}, 1)$, and the respective unit cell vectors in reciprocal space are $\mathbf{b}_{1}=\frac{2 \pi}{3 \sqrt{3} a}(-1, \sqrt{3})$ and $\mathbf{b}_{2}=\frac{2 \pi}{3 \sqrt{3} a}(1, \sqrt{3})$. The tight-binding Hamiltonian for the two cases we are interested in can be generically written as

$$
H=-t \sum_{\mathbf{r}, \delta} a_{\mathbf{r}}^{\dagger} b_{\mathbf{r}+\delta}+V \sum_{\mathbf{r}, \delta} a_{\mathbf{r}}^{\dagger} a_{\mathbf{r}} b_{\mathbf{r}+\delta}^{\dagger} b_{\mathbf{r}+\delta}+\text { H.c. },
$$

where $t$ is the nearest-neighbor hopping and $V$ the nearestneighbor Coulomb repulsion. We use standard notation where $a_{\mathbf{r}}\left(b_{\mathbf{r}}\right)$ annihilates an electron at position $\mathbf{r}$ in sublattice A (B). A mean field analysis of the nearest-neighbor Hubbard interaction in this model was studied in Refs. [17,18] to derive spontaneous breaking of time-reversal symmetry. Two $\mathcal{T}$-broken phases TI and TII were found associated with the complex NN hopping distributions shown schematically in Fig. 3. With respect to the usual tight-binding model for graphene, the TI phase is just a complex renormalization of the bare hopping plus a Kekule complex distortion. The latter breaks $\mathcal{T}$ and $\mathcal{I}$ though preserving their product. According to the analysis in Ref. [19] and as shown explicitly in [17,18], the Berry curvature is identically zero everywhere.

The bands in the TII phase have nontrivial Berry curvature. We have used these models first to establish the necessity of the connectivity of the remaining clean lattice to have an emerging metal. To this purpose, we have studied the level spacing variance of two representative Hamiltonians in the phases TI and TII when selective disorder is acting upon three of the six lattice basis, i.e., we disorder the sublattices $A_{i}$ equally, leaving the sublattices $B_{i}$ clean. This disorder distribution is equivalent to the one performed in the Haldane 


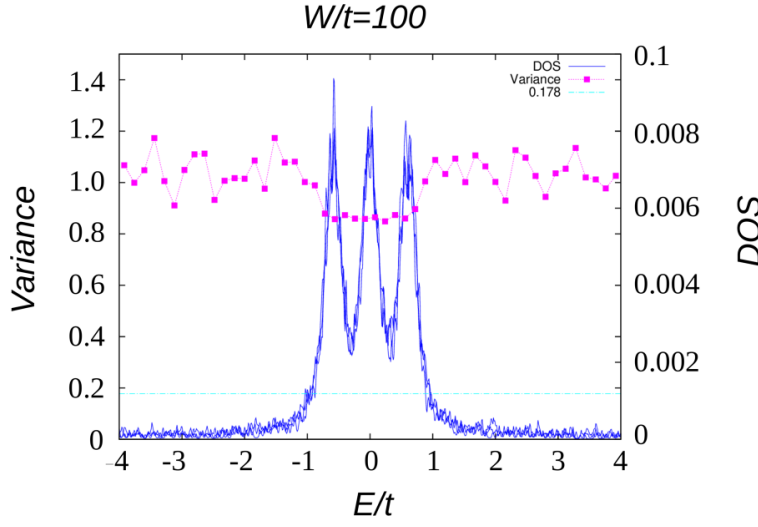

FIG. 2. Level spacing variance of the model TI with disorder distributed over three sublattices. The remaining lattice is not connected and a metallic state does not form.

model. In this case, since there is no NNN hopping parameter, the remaining clean sublattice is not connected. The level space variance of the TI model is shown in Fig. 2. As we see, all states are localized. The same occurs with the TII case.

Next we disorder one of the six sublattices, say, sublattice $A_{1}$. We use $W=100 t$. The level space variance of both models is shown in Fig. 3(c). As we can see, a metallic phase arises for strong selective disorder of one of the six sublattices. The behavior of the localization length with the size of the lattice points to the fact that, as happened in the Haldane model, the resulting metal is also a critical metal. We have checked that standard (equally distributed among the two sublattices) Anderson disorder induces normal localization in both lattices. The behavior of the TI lattice shows that a nontrivial topology is not a necessary condition to support the metallic phase. The next examples will show that the essential
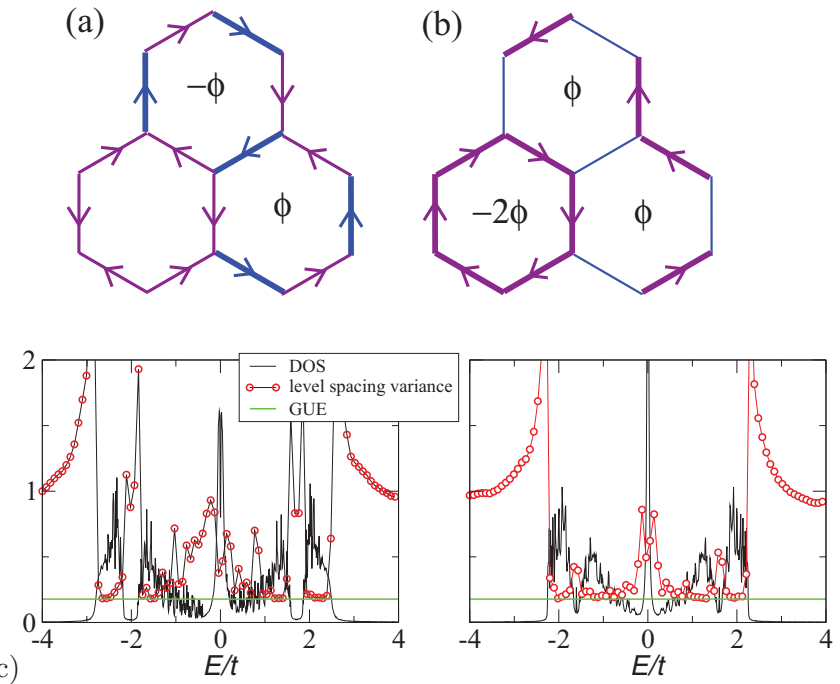

FIG. 3. Schematic representation in terms of effective hoppings and fluxes for (a) the TI phase and (b) the TII phase discussed in the text. (c) Level spacing variance of the TI and TII models showing the critical metal for strong selective disorder in one of the six sublattices. (a)

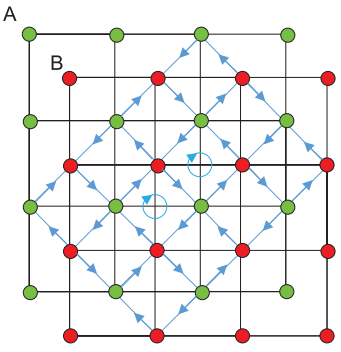

(b)
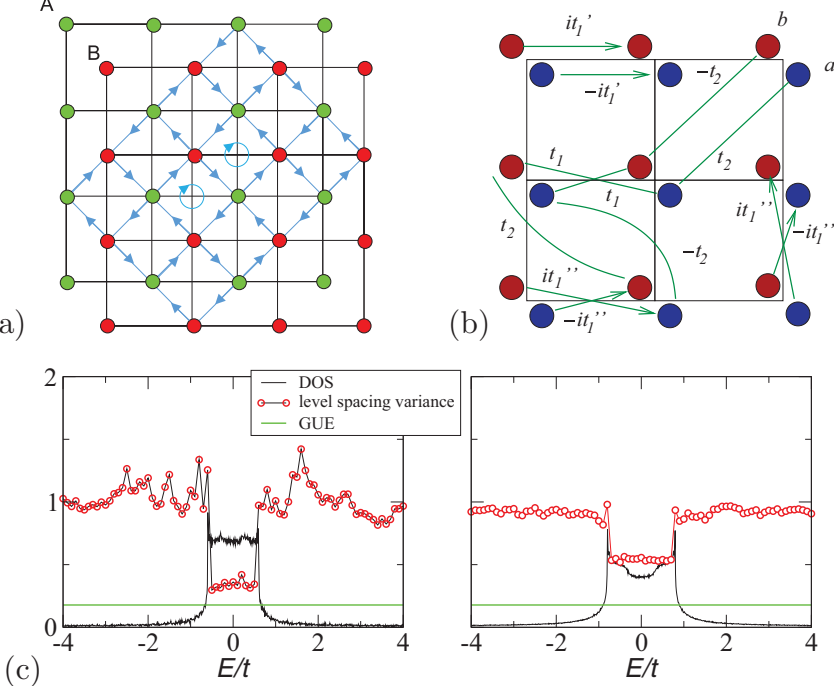

FIG. 4. Schematic representation of the hopping structure of (a) the diatomic and (b) the two-orbital model on the square lattice. (c) Level spacing variance of the lattices in (a) and (b), showing standard localization for selective disorder in only one sublattice.

property (shared by both TI and TII lattices) is the breakdown of time-reversal invariance in the clean sublattice.

Square lattice with two orbitals per site and diatomic square lattice. Our next examples of a simple Chern insulator are supported on the square lattice with two internal degrees of freedom per site that can represent orbital or spin degeneracy $s= \pm[20]$. Both tight-binding models share the property of having complex NN and real NNN hoppings. This is the reason why we have chosen them. Upon selective disorder in one sublattice, the remaining clean sublattice will have real hopping parameters and hence it will be invariant under time reversal $\mathcal{T}$. Both lattices support topologically nontrivial phases, which will be our starting point.

The Hamiltonian in both cases can be written as

$$
\begin{aligned}
H= & \sum_{\mathbf{k} \in \mathrm{BZ}} c_{\mathbf{k}}^{+} \cdot h_{\mathbf{k}} c_{\mathbf{k}}, \\
h_{\mathbf{k}}= & 2 t_{1}\left[\sin k_{x} \sigma_{x}+\sin k_{y} \sigma_{y}\right] \\
& +\left[\Delta-2 t_{2}\left(\cos k_{x}+\cos k_{y}\right)\right] \sigma_{z},
\end{aligned}
$$

where $t_{1}$ and $t_{2}$ are intraorbital and interorbital couplings, respectively, and $\Delta$ is a staggered potential. A schematic representation of the hopping structure of both lattices is shown in Figs. 4(a) and 4(b). The first example, called a diatomic square lattice $[21,22]$, is very similar to the Haldane model: The unit cell has two square plaquettes traversed by a nontrivial flux (caused in this case by the $\mathrm{NN}$ hoppings) of opposite signs so that the total flux in the Brillouin zone is zero. The second example is that of the two-orbital model on the square lattice. This has been used as an example of a simple tight-binding model supporting a Chern number bigger than one [23,24]. A schematic representation of the hoppings is shown in Fig. 4(b). In this case the fictitious flux induced by the complex hopping parameters per plaquette is zero. Although the two lattices are quite different, the remaining clean sublattices after selective disorder are identical. 
TABLE I. Summary of the examples given in the text. Here $\Omega$ means that the original lattice has nontrivial Berry curvature and $\mathcal{T}$ broken refers to the clean sublattice that remains after selective disorder.

\begin{tabular}{lccc}
\hline \hline Model & $\Omega$ & $\mathcal{T}$ broken & Metal \\
\hline Haldane & yes & yes & yes \\
TI & no & yes & yes \\
TII & yes & yes & yes \\
square biorbital & yes & no & no \\
square diatomic & yes & no & no \\
\hline \hline
\end{tabular}

We have analyzed the localization behavior of both lattices in the nontrivial topological phase under selective disorder in one sublattice only. Interestingly, despite the nontrivial topology of the bands, these lattices undergo standard Anderson localization upon selective disorder. Their level space variances are shown in Fig. 4(c). There is not any region in energy where the variance reaches the Gaussian unitary ensemble (GUE) value, so no extended state remains in the spectrum. In figures showing the level spacing variance, the horizontal green line marks the GUE variance value, 0.178, associated with the presence of extended states.

\section{DISCUSSION AND SUMMARY}

All the models discussed in this work, except the TI model on the honeycomb lattice, are examples of Chern insulators defined on partite two-dimensional lattices. The objective was to discuss the generality and robustness of the band of critical states found in the Haldane model under strong selective disorder [16]. The results are summarized in Table I. Perhaps the TI case, a topologically trivial, time-reversal broken model, is the most significant. Previous studies of localization (or rather lack thereof) established the presence and robustness of extended states carrying the Chern number in topologically nontrivial models [6,9,25-27] in class A (absence of any discrete symmetry). The presence of a robust metallic state in the TI lattice leads us to conclude that, irrespective of the topological properties of the original system (first column in Table I), the two minimal requirements for the absence of localization upon selective disorder are full connectivity and time-reversal invariance broken in the clean sublattice. The absence of localization in the presence of magnetic fields (broken time-reversal symmetry) has been analyzed at length in the literature $[7,8,28]$, but the hopping structure of the TI model shown on the Fig. 3(a) does not correspond to a magnetic field. The standard localization properties of the topologically nontrivial square lattices shown in Fig. 4 allows us to discard topology as a key point in the absence of localization. As mentioned in the Introduction, a band of critical states has been found in models with broken- $\mathcal{T}$ and spin-rotation symmetry. The models presented in this work are spin-rotation invariant (in fact, they are spinless models), but perhaps the sublattice symmetry plays a role similar to spin rotation (a $\mathcal{Z}_{2}$ symmetry in planar systems), broken by the selective disorder.

An important remark about our results refers to the precise nature of the partite lattices considered. The simplest example of a Chern insulator, the Haldane model, is defined in the honeycomb lattice which is naturally made of two interpenetrating triangular lattices. Selective disorder affects one of the sublattices. When there are internal degrees of freedom, as in the case of the two-orbital model in the square lattice, the selective disorder considered affects an entire geometrical sublattice (it destroys both orbitals in nearest-neighbor sites). Should we had considered one of the orbitals, say, $a$ [blue in Fig. 4(b)], as a sublattice, selective disorder of this particular sublattice would have led to the diatomic square lattice [Fig. 4(a)], a Chern insulator with broken $\mathcal{T}$, and no metallic state.

The findings of this work show that the role of disorder on two-dimensional systems, even if noninteracting, is still far from been fully understood. The experimental advances in optical lattice realizations can shed light on the nature of the critical metal found quite systematically in our $\mathcal{T}$-broken examples.

\section{ACKNOWLEDGMENTS}

We gratefully acknowledge Oscar Pozo for help with the figures. E.V.C. acknowledges financial support from FCT (Portugal) through Grant No. EXPL/FIS-NAN/1728/2013. This research was supported in part by the Spanish MINECO Grants No. FIS2014-57432-P and No. PGC2018-099199B-I00, the European Union Structural Funds, and the Comunidad de Madrid NMAT2D-CM Program (Grant No. S2018-NMT-4511).
[1] P. W. Anderson, Absence of diffusion in certain random lattices, Phys. Rev. 109, 1492 (1958).

[2] E. Abrahams, P. W. Anderson, D. C. Licciardello, and T. V. Ramakrishnan, Scaling Theory of Localization: Absence of Quantum Diffusion in Two Dimensions, Phys. Rev. Lett. 42, 673 (1979).

[3] A. P. Schnyder, S. Ryu, A. Furusaki, and A. W. W. Ludwig, Classification of topological insulators and superconductors in three spatial dimensions, Phys. Rev. B 78, 195125 (2008).
[4] A. MacKinnon and B. Kramer, One-Parameter Scaling of Localization Length and Conductance in Disordered Systems, Phys. Rev. Lett. 47, 1546 (1981).

[5] A. MacKinnon and B. Kramer, The scaling theory of electrons in disordered solids: Additional numerical results, Z. Phys. B 53, 1 (1983).

[6] Z. Qiao, Y. Han, L. Zhang, K. Wang, X. Deng, H. Jiang, S. A. Yang, J. Wang, and Q. Niu, Anderson Localization from the Berry-Curvature Interchange in Quantum Anomalous Hall Systems, Phys. Rev. Lett. 117, 056802 (2016). 
[7] Y. Su, C. Wang, Y. Avishai, Y. Meir, and X. R. Wang, Absence of localization in disordered two-dimensional electron gas at weak magnetic field and strong spin-orbit coupling, Sci. Rep. 6, 33304 (2016).

[8] C. Wang, Y. Su, Y. Avishai, Y. Meir, and X. R. Wang, Band of Critical States in Anderson Localization in a Strong Magnetic Field with Random Spin-Orbit Scattering, Phys. Rev. Lett. 114, 096803 (2015).

[9] Z. Xu, L. Sheng, D. Y. Xing, E. Prodan, and D. N. Sheng, Topologically protected extended states in disordered quantum spin-Hall systems without time-reversal symmetry, Phys. Rev. B 85, 075115 (2012)

[10] J. M. Kosterlitz, The critical properties of the two-dimensional xy model, J. Phys. C 7, 1046 (1974).

[11] B. Sbierski, G. Pohl, E. J. Bergholtz, and P. W. Brouwer, Quantum Transport of Disordered Weyl Semimetals at the Nodal Point, Phys. Rev. Lett. 113, 026602 (2014).

[12] C.-Z. Chen, J. Song, H. Jiang, Q.-f. Sun, Z. Wang, and X. C. Xie, Disorder and Metal-Insulator Transitions in Weyl Semimetals, Phys. Rev. Lett. 115, 246603 (2015).

[13] R. Chen, C.-Z. Chen, J.-H. Sun, B. Zhou, and D.-H. Xu, Phase diagrams of Weyl semimetals with competing intraorbital and interorbital disorders, Phys. Rev. B 97, 235109 (2018).

[14] F. D. M. Haldane, Model for a Quantum Hall Effect without Landau Levels: Condensed-Matter Realization of the Parity Anomaly, Phys. Rev. Lett. 61, 2015 (1988).

[15] E. V. Castro, M. P. López-Sancho, and M. A. H. Vozmediano, Anderson localization and topological transition in Chern insulators, Phys. Rev. B 92, 085410 (2015).

[16] E. V. Castro, R. de Gail, M. P. López-Sancho, and M. A. H. Vozmediano, Absence of localization in a class of topological systems, Phys. Rev. B 93, 245414 (2016).

[17] E. V. Castro, A. G. Grushin, B. Valenzuela, M. A. H. Vozmediano, A. Cortijo, and F. de Juan, Topological Fermi
Liquids from Coulomb Interactions in the Doped Honeycomb Lattice, Phys. Rev. Lett 107, 106402 (2011).

[18] A. G. Grushin, E. V. Castro, A. Cortijo, F. de Juan, M. A. H. Vozmediano, and B. Valenzuela, Charge instabilities and topological phases in the extended Hubbard model on the honeycomb lattice with enlarged unit cell, Phy. Rev. B 87, 085136 (2013).

[19] K. Sun and E. Fradkin, Time-reversal symmetry breaking and spontaneous anomalous Hall effect in Fermi fluids, Phys. Rev. B 78, 245122 (2008).

[20] Y.-L. Wu, B. A. Bernevig, and N. Regnault, Zoology of fractional Chern insulators, Phys. Rev. B 85, 075116 (2012).

[21] J.-M. Hou, Hidden-Symmetry-Protected Topological Semimetals on A Square Lattice, Phys. Rev. Lett. 111, 130403 (2013).

[22] B. Ostahie, M. Niţă, and A. Aldea, Edge-state mechanism for the anomalous quantum Hall effect in a diatomic square lattice, Phys. Rev. B 98, 125403 (2018).

[23] E. Prodan, T. L. Hughes, and B. A. Bernevig, Entanglement Spectrum of a Disordered Topological Chern Insulator, Phys. Rev. Lett. 105, 115501 (2010).

[24] A. G. Grushin, T. Neupert, C. Chamon, and C. Mudry, Enhancing the stability of a fractional Chern insulator against competing phases, Phys. Rev. B 86, 205125 (2012).

[25] A. Pruisken, The integral quantum Hall effect: Shortcomings of conventional localization theory, Nucl. Phys. B 295, 653 (1988).

[26] A. W. W. Ludwig, M. P. A. Fisher, R. Shankar, and G. Grinstein, Integer quantum Hall transition: An alternative approach and exact results, Phys. Rev. B 50, 7526 (1994).

[27] M. A. Werner, A. Brataas, F. von Oppen, and G. Zaránd, Anderson localization and quantum Hall effect: Numerical observation of two-parameter scaling, Phys. Rev. B 91, 125418 (2015).

[28] P. Kleinert and V. V. Bryksin, Microscopic theory of Anderson localization in a magnetic field, Phys. Rev. B 55, 1469 (1997). 